\title{
Chondroitinase ABC Digestion of the Perineuronal Net Promotes Functional Collateral Sprouting in the Cuneate Nucleus after Cervical Spinal Cord Injury
}

\author{
James M. Massey, ${ }^{1,2,3,4}$ Charles H. Hubscher, ${ }^{2,4}$ Michelle R. Wagoner, ${ }^{3,4}$ Julie A. Decker, ${ }^{3,4}$ Jeremy Amps, ${ }^{5}$ Jerry Silver, ${ }^{5}$ \\ and Stephen M. Onifer ${ }^{2,3,4}$ \\ ${ }^{1} \mathrm{MD} / \mathrm{PhD}$ Program, Departments of ${ }^{2}$ Anatomical Sciences and Neurobiology and ${ }^{3}$ Neurological Surgery, and ${ }^{4}$ Kentucky Spinal Cord Injury Research Center, \\ School of Medicine, University of Louisville, Louisville, Kentucky 40292, and ${ }^{5}$ Department of Neurosciences, Case Western Reserve University School of \\ Medicine, Cleveland, Ohio 44106
}

\begin{abstract}
Upregulation of extracellular chondroitin sulfate proteoglycans (CSPGs) after CNS injuries contributes to the impediment of functional recovery by restricting both axonal regeneration and synaptic plasticity. In the present study, the effect of degrading CSPGs with the application of the bacterial enzyme chondroitinase $\mathrm{ABC}(\mathrm{chABC})$ into the cuneate nucleus of rats partially denervated of forepaw dorsal column axons was examined. A dorsal column transection between the $\mathrm{C} 6-\mathrm{C} 7$ dorsal root entry zones was followed immediately by an ipsilateral brainstem injection of either chABC or a bacterial-derived control enzyme [penicillinase (P-ase)] and then subsequently $(1$ week later) followed with a second brainstem enzyme injection and cholera toxin B subunit (CTB) tracer injection into the ipsilateral forepaw digits and pads. After 1 additional week, the rats underwent electrophysiological receptive field mapping of the cuneate nucleus and/or anatomical evaluation. Examination of the brainstems of rats from each group revealed that CSPGs had been reduced after chABC treatment. Importantly, in the chABC-treated rats (but not in the P-ase controls), a significantly greater area of the cuneate nucleus was occupied by physiologically active CTB traced forepaw afferents that had been spared by the initial cord lesion. These results demonstrate, for the first time, a functional change directly linked to anatomical evidence of sprouting by spinal cord afferents after chABC treatment.
\end{abstract}

Key words: somatosensory system; proteoglycan; axonal regeneration; synaptic plasticity; synaptogenesis; extracellular matrix; receptive field; microelectrode; dorsal columns

\section{Introduction}

Spinal cord injury (SCI) results in sensory and motor deficits by depriving synaptically linked neurons of their afferent or efferent inputs. Upregulation of extracellular chondroitin sulfate proteoglycans (CSPGs) that takes place at various times after CNS injuries is a major factor that contributes to regeneration failure (McKeon et al., 1991; Pindzola et al., 1993; Davies et al., 1999; Jones et al., 2003; Tang et al., 2003; Silver and Miller, 2004). The development of strategies that manipulate the production of these inhibitory molecules or alter the response of the regenerating axon to them are the current focus of intense investigations

\footnotetext{
Received Dec. 21, 2005; revised Feb. 21, 2006; accepted March 19, 2006.

This work was supported by National Institutes of Health Grants NS40411 and RR15576 (S.M.0.) and 25713 (J.S.) J.M.M. was supported by a fellowship awarded from a Kentucky National Science Foundation/Experimental Program to Stimulate Competitive Research Grant EPS-9874764 (N. G. F. Cooper). We thank Russell T. Matthews (Yale University, New Haven, (T) for assistance with immunohistochemistry, Robin Krimm (University of Louisville, Louisville, $\mathrm{KY}$ ) for the generous donation of the Dil used in the electrophysiological experiments, and George Harding for his help with confocal microscopy. We are grateful to Christine Nunn, Christopher Whitaker, Catherine Doller, Jim Armstrong, and Mike Lupa for technical assistance. We also thank Aaron Puckett and the staff at the University of Louisville Research Resources Center for assistance with veterinary care.

Correspondence should be addressed to Dr. Stephen M. Onifer, Spinal Cord and Brain Injury Research Center University of Kentucky, Biomedical and Biological Sciences Research Building B365, 741 South Limestone Street, Lexington, KY 40536-0509. E-mail: stephen.onifer@uky.edu.

DOI:10.1523/JNEUROSCI.5467-05.2006

Copyright $\odot 2006$ Society for Neuroscience $\quad$ 0270-6474/06/264406-09\$15.00/0
}

(Filbin, 2003; Silver and Miller, 2004). One particularly effective strategy is the removal of glycosaminoglycan side chains from the proteoglycan core protein with the bacterial enzyme chondroitinase $\mathrm{ABC}$ (chABC). Used alone or in concert with other treatments, chABC application has led to several reports of axon regeneration and functional recovery (McKeon et al., 1991, 1999; Yick et al., 2000, 2003; Moon et al., 2001; Bradbury et al., 2002; Chau et al., 2004; Caggiano et al., 2005; Fouad et al., 2005; Steinmetz et al., 2005).

CSPGs are also major components of the extracellular matrix found throughout the normal adult CNS (Steindler, 1993). Some proteoglycan family members participate in the formation of the so-called perineuronal net, a lace-like structure (TakahashiIwanaga et al., 1998) produced by both neurons and astrocytes that encapsulates synapses throughout the nervous system (Golgi, 1893). An emerging body of evidence indicates that the normal expression of CSPGs surrounding neuronal membranes plays a critical role in the regulation of axon terminal arbor sprouting during development and after denervating injuries. Expression of CSPGs is low during the initial stages of synaptogenesis, and their increase coincides with the end of critical period plasticity (Lander et al., 1997; Pizzorusso et al., 2002). This increase is dependent on normal activity in both the brain and spinal cord (Kalb and Hockfield, 1990a,b; Lander et al., 1997; 

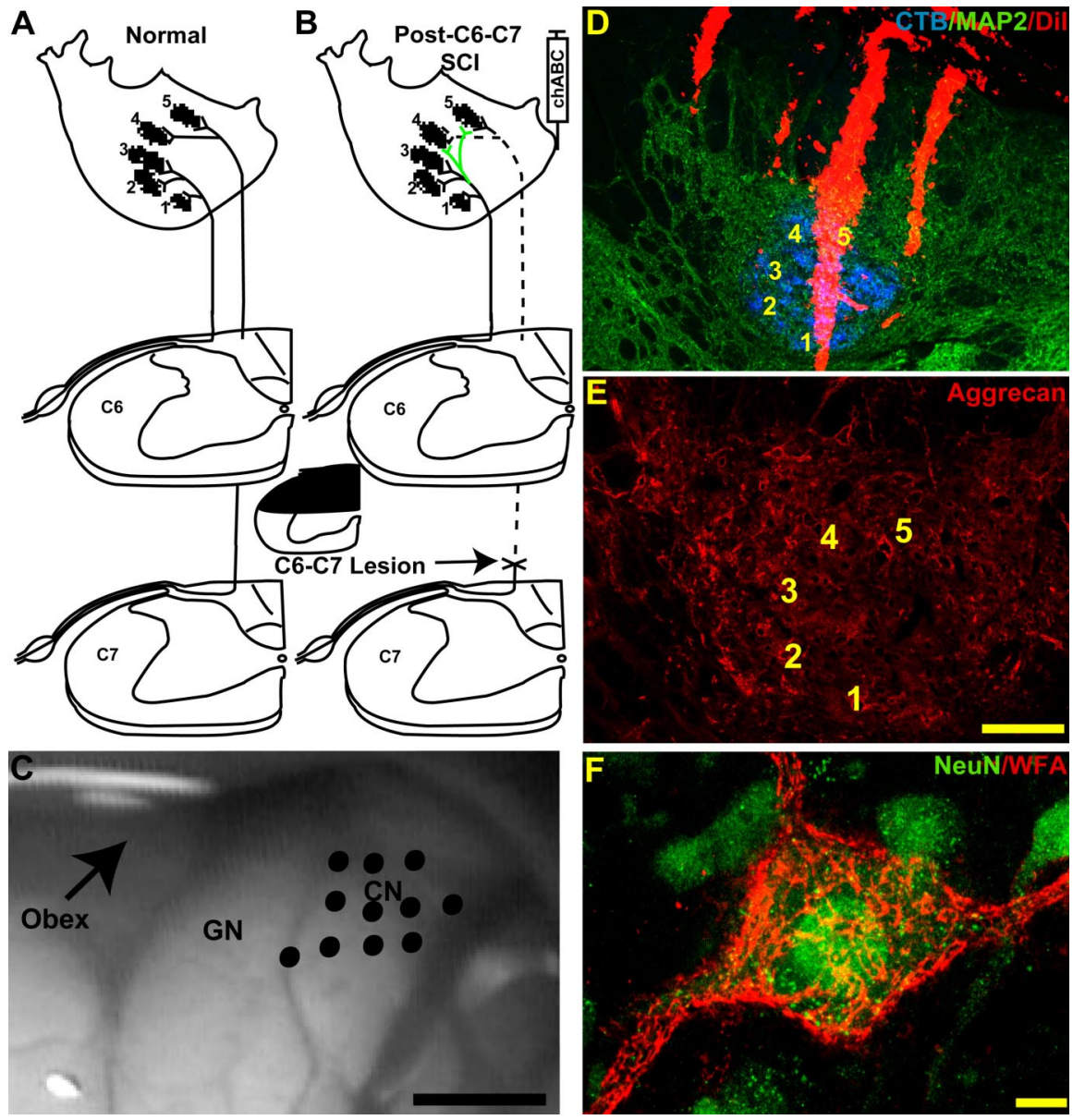

Figure 1. Diagram summary of the experimental design. $\boldsymbol{A}$, Normal forepaw primary afferents in the rat cuneate nuclei are distributed in a somatotopic arrangement. $\boldsymbol{B}$, To assess the effect of chABC digestion on collateral sprouting in the cuneate nuclei, the dorsal columns were transected between the $C 6$ and $C 7$ root entry zones denervating all primary afferent input from digits 4 and 5 plus most of the input from digit 3. chABC was then injected adjacent to the ipsilateral cuneate nuclei. Microelectrode receptive field mapping was used in combination with CTB injections into the palmar digits and footpads to demonstrate the functional and anatomical distribution of forepaw primary afferent terminals. $\boldsymbol{C}$, Image of the exposed brainstem showing the dorsal surface of the dorsal column nuclei from a sham rat that underwent electrophysiological assessment. Electrode entry points into the cuneate nuclei (CN) are marked by the black dots. GN, Gracile nucleus. D, Each electrode was coated with Dil (red), which allowed anatomical localization of the recording penetrations in transverse sections labeled for cuneate nuclei neurons and primary afferent anatomy by MAP2-IR (green) and CTB-IR (blue). E, Aggrecan-IR using the Cat-301 antibody labels perineuronal nets and extracellular matrix in the normal cuneate nuclei. $\boldsymbol{F}$, Using high-power confocal microscopy, CSPGs contained in the perineuronal net labeled by WFA cytochemistry (red) form a lace-like cloak around NeuN-immunoreactive (green) cuneate nuclei neurons. Numbers $1-5$ denote the normal locations of the digit terminal fields. Scale bars: $\boldsymbol{C}, 1 \mathrm{~mm} ; \boldsymbol{D}, 200 \mu \mathrm{m} ; \boldsymbol{E}, 100 \mu \mathrm{m}$; $\boldsymbol{F}, 10 \mu \mathrm{m}$.

Pizzorusso et al., 2002) and mediated through activation of NMDA glutamate receptors (Kalb and Hockfield, 1990b). Enzymatic digestion by chABC collapses much of the net-associated extracellular matrix of cortical layers (Bruckner et al., 1998; Pizzorusso et al., 2002) and leads to reactivation of plasticity in normal adult animals (Pizzorusso et al., 2002; Corvetti and Rossi, 2005). chABC applied to lesion-denervated targets has also been shown to enhance collateral sprouting and synapse formation by spared fibers (Tropea et al., 2003).

Collateral sprouting by intact primary afferents has been shown to be extremely limited within the adult rat dorsal column nuclei denervated by SCI (Jain et al., 1995). In recent experiments, we observed that CSPGs are expressed in the normal dorsal column nuclei and that they are significantly increased after SCI (Massey et al., 2005). In the present study, anterograde anatomical tracing and microelectrode receptive field mapping were used to determine whether chABC application promotes collateral sprouting by spared forepaw primary afferents within the cuneate nucleus partially denervated by a C6-C7 dorsal column transection.

\section{Materials and Methods}

All procedures complied with National Institutes of Health and University of Louisville Institutional Animal Care and Use Committee guidelines. For each surgical procedure and electrophysiological assessment, the 19 adult male (250-300 g) Sprague Dawley rats were anesthetized with intramuscular injections of a mixture of ketamine hydrochloride $(50 \mathrm{mg} /$ $\mathrm{kg})$, xylazine $(6.5 \mathrm{mg} / \mathrm{kg})$, and acepromazine (2.5 mg/kg).

Cervical SCI. Two experimenters performed the surgical procedures. Age-matched rats were randomly assigned by one experimenter into the experimental groups. The dorsal portions of the $\mathrm{C} 5$ and $\mathrm{C} 6$ vertebrae were removed, exposing the $\mathrm{C} 6$ and $\mathrm{C} 7$ dorsal root entry zones. A 1.5-mmdeep dorsal-to-ventral laceration was made with small, depth-marked spring scissors between the caudal C6 and rostral C7 dorsal rootlets from the lateral portion of the right side of the dorsal spinal cord, through the right dorsal horn and into the left gracile fasciculus (Fig. $1 A, B$ ). Each lesion was verified for completeness by macroscopic inspection (for level) and microscopic examination (for depth, i.e., through the central canal) by the second experimenter.

Enzyme delivery and primary afferent tracing. To assess the acute effects of chABC digestion on brainstem anatomy, three normal rats received bilateral medulla injections of either $1 \mu \mathrm{l}$ of $50 \mathrm{U} / \mathrm{ml}$ penicillinase (P-ase) $(n=1$; Sigma, St. Louis, $\mathrm{MO}$ ) or $1 \mu \mathrm{l}$ of $50 \mathrm{U} / \mathrm{ml}$ protease-free $\operatorname{chABC}(n=2$; Seikagaku America, Falmouth, $\mathrm{MA}$ ) and were killed $1 \mathrm{~d}$ later. There were no obvious gross effects on brain tissue after either injection. In animals receiving enzyme treatments after SCI, the right medulla was exposed immediately after the injury, and $1 \mu \mathrm{l}$ of 50 $\mathrm{U} / \mathrm{ml} \mathrm{P}$-ase $(n=5)$ or chABC $(n=6)$ was injected at $0.5-0.3 \mathrm{~mm}$ below the dorsal pial surface and just lateral to the cuneate nucleus (Fig. 1B) using a mechanical microinjector (Nanoject II; Drummond Scientific, Broomall, PA). P-ase is an especially good control because it is a bacterial-derived enzyme with a similar molecular weight to chABC and it has been shown to have no effect on CNS tissue (Moon et al., 2001; Bradbury et al., 2002; Pizzorusso et al., 2002; Caggiano et al., 2005). One week after receiving SCI and enzyme injection, the right brainstem of each rat was reexposed, and a second injection of either $\mathrm{P}$-ase or chABC was made as described above to further digest any newly acquired CSPGs (Massey et al., 2005). To visualize the central terminals of cutaneous right forepaw primary afferents, 2-4 $\mu \mathrm{l}$ of $1 \%$ cholera toxin B subunit (CTB) (Sigma) was injected subcutaneously into the palmar side of each digit and into the glabrous footpad (Onifer et al., 2005) at this time. One week later and, thus, 2 weeks after the spinal cord injury, rats from both groups underwent terminal electrophysiological evaluation and/or were killed for anatomical evaluation. Sham control rats $(n=5)$ had their brainstems and spinal cords exposed but did not receive a SCI or enzyme injections. They received CTB tracing and then either underwent the terminal electrophysiological evaluation or were killed for anatomy 1 week later. 
Microelectrode receptive field mapping of the cuneate nucleus. Four $\mathrm{P}$-ase-treated injured rats, four chABC-treated injured rats and 2 sham control rats underwent microelectrode receptive field mapping. Animals were anesthetized and then placed in a stereotaxic device (model 900; David Kopf Instruments, Tujunga, CA), and subsequent injections of anesthesia were given as needed approximately every 45-60 min. Multiunit and occasional single-unit responses to cutaneous stimuli were recorded using a tungsten microelectrode (impedance of 6-8 $\mathrm{M} \Omega$; Frederick Haer Company, Bowdoinham, ME) (Hubscher and Berkley, 1994). To visualize the recording tracks, 3-5 $\mathrm{ml}$ of DiI (Invitrogen, Carlsbad, CA) were applied to each electrode and allowed to dry overnight before each experiment. Rows of recording tracks were made through the cuneate nucleus perpendicular to the pial surface. Each row was spaced 200 $\mu \mathrm{m}$ apart in the rostral-to-caudal plane. The recording track within each row was separated by $100-200 \mu \mathrm{m}$ (Fig. $1 C$ ). The electrode track entry points were marked on digital photos taken of the dorsal surface of each exposed brainstem (Fig. 1C). The electrode was advanced in $50 \mu \mathrm{m}$ intervals using a hydraulic microdrive unit (Frederick Haer Company). Low-threshold cutaneous stimuli were delivered to the ipsilateral forepaw and the entire body surface at each recording site, to identify the complete extent of each receptive field, by light touch using either a small brush or Semmes-Weinstein monofilaments (North Coast Medical, Morgan Hill, CA). Responses to high-threshold stimuli (movement/joint pressure) were distinguished from cutaneous stimuli and were not used for subsequent quantification. Noxious stimuli were not used. The stimulated areas of the body eliciting a response were recorded by a second experimenter, and outlines were drawn onto scaled rat body maps. Each rat was killed at the end of this procedure.

Histology. After an overdose of sodium pentobarbital (120 mg/kg, i.p.), transcardiac perfusions of each rat were performed with oxygenated and heparinized calcium-free Tyrode's solution and then with $4 \%$ paraformaldehyde in 0.1 m phosphate buffer (PB), pH 7.4 (Onifer et al., 1993). The cervical spinal cord and brainstem were removed and cryoprotected in 30\% sucrose/PB for 3-4 d. The tissues from animals that underwent electrophysiology or used for terminal field quantification were sectioned in the transverse plane at $20 \mu \mathrm{m}$ with a cryostat. Tissues from normal animals used for visualization of perineuronal nets were sectioned at $30 \mu \mathrm{m}$. All sections were then mounted onto charged microscope slides and stored at $-80^{\circ} \mathrm{C}$. The spinal cord and brainstem sections were incubated with primary antibodies diluted in Tris-buffered saline, pH 7.4, containing $0.25 \%$ Triton X-100 (Sigma) and 5\% normal donkey serum (Jackson ImmunoResearch, West Grove, PA). A combination of mouse anti-microtubule associated peptide $2 \mathrm{a}+2 \mathrm{~b}$ (MAP2) (1:200; Sigma), neuron-specific nuclear protein (NeuN) (1:500; Chemicon, Temecula, CA), and goat anti-CTB (1:1000; List Biological Laboratories, Hornby, Ontario, Canada) were used to visualize the anatomical features of the cuneate nucleus and to label CTB traced cutaneous forepaw primary afferents, respectively. We have shown that MAP2 immunoreactivity (IR) reliably labels both the borders and the anatomical architecture of the normal and denervated adult rat cuneate nucleus (Massey et al., 2003). To test the effectiveness of chABC treatment, brainstem sections were processed for Wisteria floribunda agglutinin (WFA) cytochemistry (as described by Bruckner et al., 1998) to see perineuronal nets or were reacted with mouse antibodies that recognize $\mathrm{C} 4$ sulfated glycosaminoglycan "stubs" (2B6; 1:200; Seikagaku America) (Bruckner et al., 1998), full-length and N-terminal neurocan (1F6; 1:200; Developmental Studies Hybridoma Bank, University of Iowa, Iowa City, IA) (MeyerPuttlitz et al., 1995), or CNS aggrecan (Cat-301; 1:200; Dr. Russell T. Matthews, Yale University, New Haven, CT) (Matthews et al., 2002). After incubation with the primary antibody mixture, the sections were reacted with the appropriate species-specific donkey secondary antibodies conjugated with fluorescein isothiocyanate, cyanine 3 , or 7-amino-4-methyl-3-coumarinylacetic acid (Jackson ImmunoResearch). The sections were coverslipped and images were obtained using a Nikon (Tokyo, Japan) Eclipse E400 microscope connected to a SPOT Insight 3.2 color camera.

Quantification of CTB-labeled terminal fields. Using an Olympus Optical (Melville, NY) 3 Laser Fluoview 500 scanning confocal microscope, $40 \times$ confocal images of the cuneate nuclei were obtained from serial sections at $200 \mu \mathrm{m}$ intervals beginning $300 \mu \mathrm{m}$ caudal to the obex (Maslany et al., 1992). Sections from these locations were chosen because they were at the maximal distance $(100 \mu \mathrm{m})$ identified to contain microelectrode recording tracks. Sections from each P-ase-treated $(n=5)$ or chABC-treated $(n=6)$ injured rat were analyzed. The photomultiplier settings were identical for each section. Each confocal image was then given a code identifier and analyzed by a blinded experimenter using Matlab (version 6.5; MathWorks, Natick, MA) and its corresponding Image Processing Toolbox. A low-threshold criterion was selected to remove background fluorescence and was applied in a nonbiased manner to all images. All pixels above this value were counted, and their total area was calculated. Mean values of the CTB-labeled terminal areas from P-ase-treated rats $(n=5)$ and chABC-treated rats $(n=6)$ were compared using the Student's $t$ test of the SPSS 13.0 and SigmaPlot 8.0 statistical software (SPSS, Chicago, IL).

Receptive field reconstructions and quantification. The DiI-labeled electrode tracks in the brainstems were identified, and images were captured using the Nikon Eclipse E400 microscope connected to a SPOT Insight 3.2 color camera. The sections containing recording tracks were then processed for MAP2- and CTB-IR. Locations of recording tracks were matched to the digitized images of the DiI-labeled recording tracks. Only recording tracks verified to be within the cuneate nucleus were used for quantification. A total of 964 confirmed recording sites were obtained from both the right and left cuneate nuclei of two sham rats $(n=492)$ and from the right cuneate nuclei of both the P-ase-treated $(n=204)$ and chABC-treated $(n=268)$ injured rats. The frequencies at which the recording sites within the cuneate nuclei were responsive to stimulation of the ipsilateral forepaw or other body surfaces or were unresponsive to any stimuli were determined for each rat, and then a mean value was generated for each group. These values were compared using one-way ANOVA and Tukey's post hoc pairwise comparisons. The frequency of individual digit responses recorded from each rat was also averaged among groups. These data were compared by two-way ANOVA and Tukey's post hoc pairwise comparisons. All statistical analyses were performed using SPSS 13.0 and SigmaPlot 8.0 statistical software.

\section{Results}

Denervation of the adult rat cuneate nucleus by SCI (Fig. 1B) results in a reactive gliosis and upregulation in associated CSPGs (Massey et al., 2005). This, combined with the CSPGs normally present in the perineuronal nets and extracellular matrix of the adult cuneate nucleus (Fig. $1 E, F$ ), constitutes a potential barrier to reactive collateral sprouting (Tropea et al., 2003).

\section{ch $A B C$ degrades glycosaminoglycan side chains in the nucleus cuneatus}

Cutaneous CTB injections into the forepaw digits and volar pads of control rats resulted in labeled primary afferent terminals within the cuneate nucleus (Onifer et al., 2005) that were distributed in a precise, somatotopic pattern as has been described previously (Maslany et al., 1991, 1992). These terminals associate with dense clusters of recipient MAP2-immunoreactive cuneate neuron dendrites (Fig. 1D) (Massey et al., 2003).

To confirm that chABC deglycosylates endogenous CSPGs, normal rats were killed within $1 \mathrm{~d}$ of bilateral injections of either $\mathrm{P}$-ase or chABC into the caudal brainstem. Sections containing the cuneate nuclei were processed for the intact glycosaminoglycan side chains with WFA cytochemistry or immunodetection of the 2B6 stub antigen. Structures throughout the dorsal column nuclei and other areas of the brainstem in chABC-treated rats were robustly labeled by the $2 \mathrm{~B} 6$ antibody (Fig. $2 A-C, E$ ). A border between 2B6-IR and WFA-labeled areas of the brainstem clearly demonstrated the extent of chABC digestion (Fig. 2E). 2B6-IR extended radially for $>8 \mathrm{~mm}^{3}$ from the point of injection. No 2B6-IR was observed in the brainstems of sham control or P-ase-treated rats (Fig. $3 B$ ). 

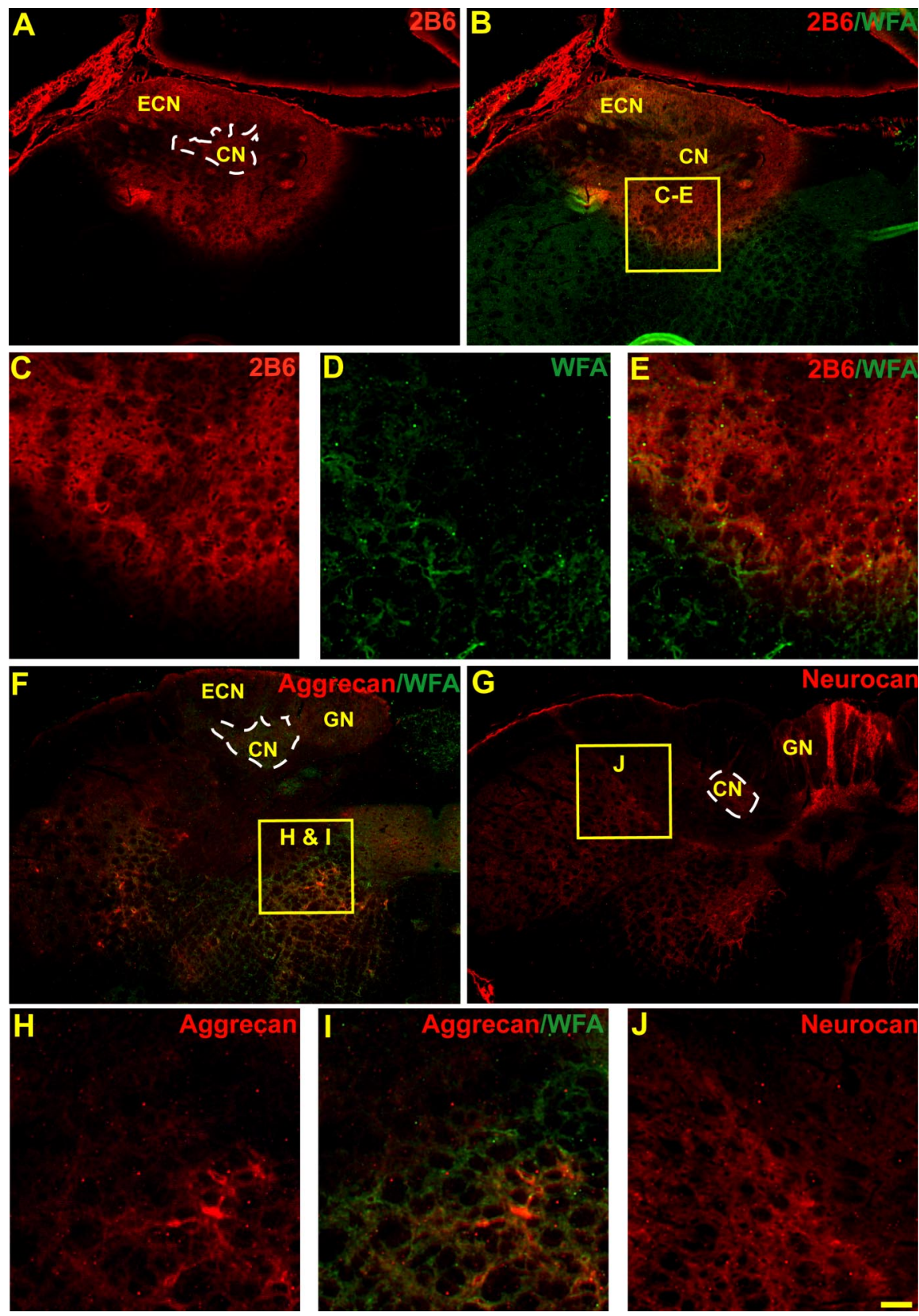

Figure 2. Sections through a normal rat brainstem demonstrating the effectiveness of chABC injections. chABC effectively reduces glycosaminoglycans on CSPGs in the normal cuneate nucleus (CN) and nearby areas of the medulla after in vivo injections into the caudal brainstem. WFA (green) and 2B6 (stub antibody, red) label complementary areas and demonstrate the extent of chABC digestion of glycosaminoglycan moieties $(\boldsymbol{A}-\boldsymbol{E})$. Both aggrecan (Cat-301; red, $\boldsymbol{F}, \boldsymbol{H}, \boldsymbol{I}$; compare with Fig. $1 E$ ) and neurocan (1F6; red, $\mathbf{G}, \boldsymbol{J}$ ) core protein immunoreactivity was also reduced in areas heavily labeled by $2 \mathrm{~B} 6$ immunoreactivity in adjacent sections. At higher power, borders of glycosaminoglycan chain digestion (absence of green WFA staining) next to intact CSPGs (presence of WFA green staining) shared similar borders with the reduction of CSPG core proteins $(\boldsymbol{H}, \boldsymbol{I})$. The dotted white line demonstrates the area of the cuneate nucleus $(\boldsymbol{A}, \boldsymbol{F}, \boldsymbol{G})$. ECN, External cuneate nucleus; $G N$, gracile nucleus. Scale bar: $\boldsymbol{A}, \boldsymbol{B}, \boldsymbol{F}, \boldsymbol{G}, 200$ $\mu \mathrm{m} ; \boldsymbol{C}-\boldsymbol{E}, \boldsymbol{H}-\boldsymbol{J}, 60 \mu \mathrm{m}$.

Immunolabeling by antibodies to both neurocan (1F6) and aggrecan (Cat301) was also reduced by chABC treatment in areas lacking WFA labeling or densely labeled by 2B6-IR in adjacent brainstem sections (Fig. $2 \mathrm{~F}-J$ ). Similar reductions in perineuronal net CSPG staining have been reported previously after chABC injections in the adult rat cortex (Bruckner et al., 1998; Pizzorusso et al., 2002) and cerebellum (Corvetti and Rossi, 2005), and now we show this effect in the brainstem. The reason for, and the physiological consequence of, the apparent loss of CSPG core protein antigenicity after enzyme treatment is unclear. Importantly, these results do indicate that chABC administration is effective at diminishing the potential inhibitory influence of CSPGs and their side chains, known to be inhibitory to dorsal root ganglion axon extension (Snow et al., 1990).

We found a dramatic increase in CSPG expression in the adult rat dorsal column nuclei during the first 3 weeks after cervical SCI (Massey et al., 2005). Because of this, a second injection of chABC or P-ase was given 1 week after the spinal cord injuries. Examination of the brainstems from these rats revealed the same pattern of 2B6-IR observed in the acutely chABCtreated normal rats (Fig. $3 A$ ). Thus, acute chABC administration after SCI combined with a second injection 1 week later effectively modified CSPGs in the cuneate nucleus.

\section{chABC treatment promotes collateral sprouting of remaining forepaw primary afferent terminals}

We reported previously that C4 spinal cord dorsal column transection anatomically and electrophysiologically denervates the cuneate nuclei (Onifer et al., 2005). In pilot experiments, we determined that transecting dorsal root axons just caudal to the C6 spinal cord level resulted in a substantial decrease of CTB traced fibers leaving labeled terminals primarily in areas corresponding to digits 1 and 2 and a small portion of the dorsal cuneate nucleus (our unpublished observations). In the $\mathrm{P}$-ase-treated injured rats, much of the CTB-immunoreactive traced forepaw primary afferent terminals in the cuneate nuclei were observed in the expected somatotopic locations of digits 1 and 2 (Fig. 4A,B). Occasionally, sparse CTB-IR was also observed in the ventral portion of the digit 3 representation and along the dorsal edge of the cuneate nucleus near the areas occupied by dorsal digit afferents (Maslany et al., 1991). Only very infrequently were sparse collections of randomly distributed terminals observed well outside their normal somatotopic positions.

When the brainstems of chABCtreated injured rats were examined, the CTB-immunoreactive $\left(323.73 \pm 69.53 \mu \mathrm{m}^{2}\right)$ forepaw terminal area was significantly greater compared with the P-ase-treated $\left(165.41 \pm 67.63 \mu \mathrm{m}^{2}\right.$; Student's $t$ test, $p<0.01$ ) injured rats (Fig. 4). Although some of this difference could be attributed to a higher density of CTBimmunoreactive terminals located within the proper terminal fields of digits 1 and 2, much of the increased CTB-IR could be seen in ectopic areas (i.e., those normally innervated by the other digits and other forepaw and forelimb surfaces). In contrast to the 


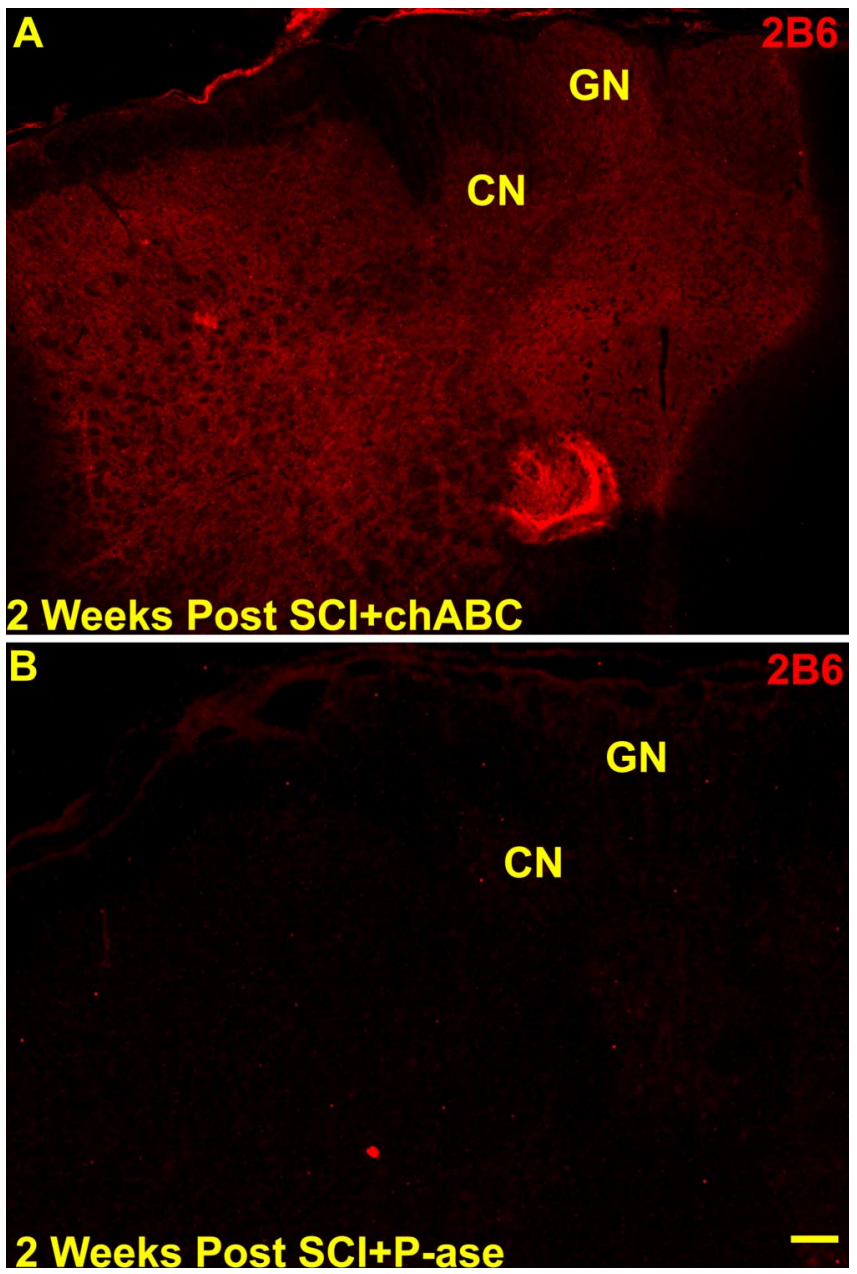

Figure 3. After a $(6-C 7 S C l$ and two injections of either $\operatorname{ch} A B C(A)$ or $P$-ase ( $B$; at the time of cord injury and 1 week later), evidence of enzyme digestion demonstrated by 2B6immunoreactive labeling of ( 4 sulfated glycosaminoglycan "stubs" similar to that seen in normal rats (see Fig. 2) was observed in chABC-treated $(\boldsymbol{A})$ but not in P-ase-treated $(\boldsymbol{B})$ injured rats. CN, Cuneate nucleus; GN, gracile nucleus. Scale bar, $200 \mu \mathrm{m}$.

CTB-immunoreactive terminals observed in sham and P-asetreated injured rats, divisions between adjacent digit terminal zones were often less distinct, and the terminal fields in some rats appeared to form a continuous band that extended along the lateral edge, through adjacent digit representation areas and into the dorsal portions of the cuneate nucleus (Fig. 4C,D). These results indicate that chABC promotes sprouting of uninjured forelimb primary afferents into denervated areas of the cuneate nucleus after cervical SCI.

\section{chABC treatment increases cuneate nuclei activation after C6-C7 SCI}

Anatomical reconstruction of the microelectrode tracks through the cuneate nuclei of the sham control rats revealed an orderly somatotopic progression of receptive fields over the surfaces of the ipsilateral forepaw, arm and trunk, and neck (Fig. 5A, B). In all sites confirmed to be located within the cuneate nucleus, low levels of synchronous background activity were observed. Shifts in the frequency of these spontaneous bursts changed with movements of as little as $50 \mu \mathrm{m}$ in electrode position and were often accompanied by shifts in receptive fields. Multiunit and occasional single-unit responses to low-threshold stimuli could be clearly distinguished in most of the cuneate nucleus and were

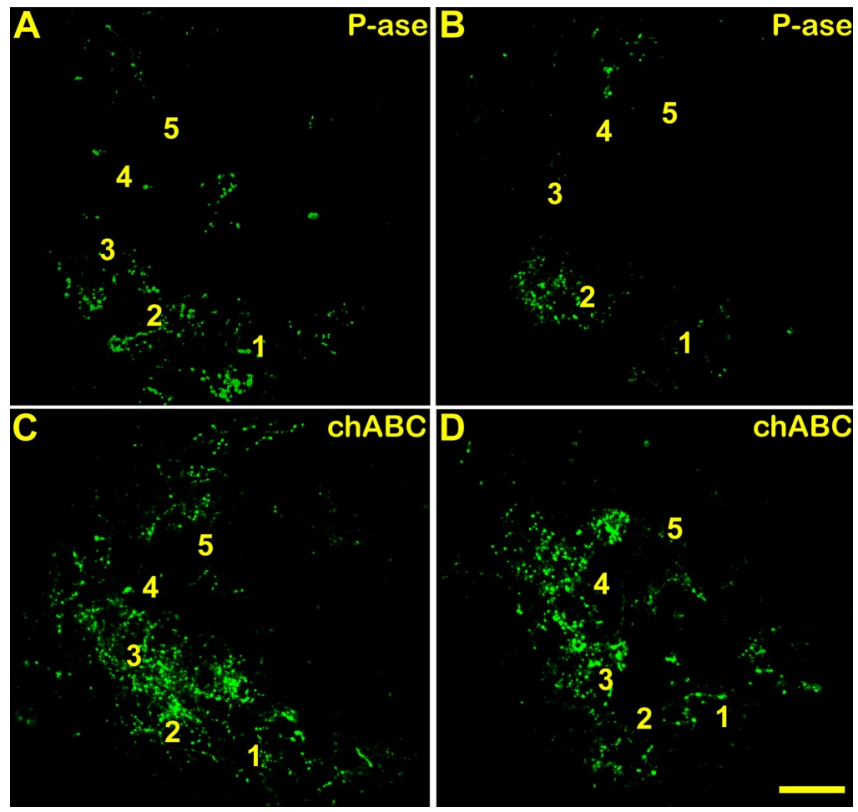

Figure 4. Representative confocal images of CTB traced forepaw primary afferent terminal fields in the cuneate nuclei of two P-ase-treated or two chABC-treated rats after $C 6-C 7$ spinal cord injuries $(\boldsymbol{A}-\boldsymbol{D})$. The CTB-IR remaining in the $P$-ase-treated rats was primarily confined to the normal somatotopic locations of digits 1 and $2(\boldsymbol{A}, \boldsymbol{B})$. This coincided with the receptive fields observed in rats that underwent electrophysiological assessment (see Figs. 5, 6). In chABCtreated injured rats, the CTB-IR was significantly more extensive than that observed in injured rats treated with $P$-ase $(\boldsymbol{E})$, spreading throughout adjacent digit locations and often well into the dorsalmost surface of the cuneate nuclei $(\boldsymbol{C}, \boldsymbol{D})$. Numbers $1-5$ denote the normal locations of the digit terminal fields. Scale bar, $50 \mu \mathrm{m}$.

often more than three times background. Large continuous receptive fields extending from the midshoulder to midthoracic areas of the trunk were observed in recording tracks through the dorsomedial portion of the cuneate nucleus (Fig. 5B). Recording tracks matched to areas densely labeled by CTB tracing of the forepaw revealed responses to areas of the digits and forepaw surface that generally corresponded to the appropriate anatomical locations (Fig. 5A,B). In rare instances, cells that only responded to deep stimuli or to directionally dependent stimuli were also observed. Portions of electrode tracks matched to areas of the external cuneate were often only responsive to movement. Only responses to cutaneous stimuli were quantified. Quantitative analysis revealed no difference in the frequency of digit receptive fields between digits in the sham control rats (Fig. 6C). Few recording sites were encountered in which no receptive fields could be mapped (Fig. 6B).

A much more limited set of receptive fields was encountered in injured rats receiving $\mathrm{P}$-ase injections. In recording tracks through the areas of the medial-to-lateral portion of the cuneate nucleus, which in sham control rats contained large receptive fields of the shoulder and midthoracic trunk, only responses to the ipsilateral shoulder could be evoked (Fig. 5C,D). Furthermore, progressions of receptive fields across the digits were no longer observed at recording sites matched to areas normally highly responsive to stimulation of the forepaw. Instead, the receptive fields were restricted to the surfaces of digits 1 and 2 and, infrequently, to a small portion of digit 3 (Figs. 5D, 6C). These sites could often be matched to digits 1,2 , and 3 areas labeled by CTB-immunoreactive terminals (Fig. 5C). Receptive fields mapped to the rest of the forepaw and forearm were similarly constrained and did not progress caudal to the dermatomes con- 

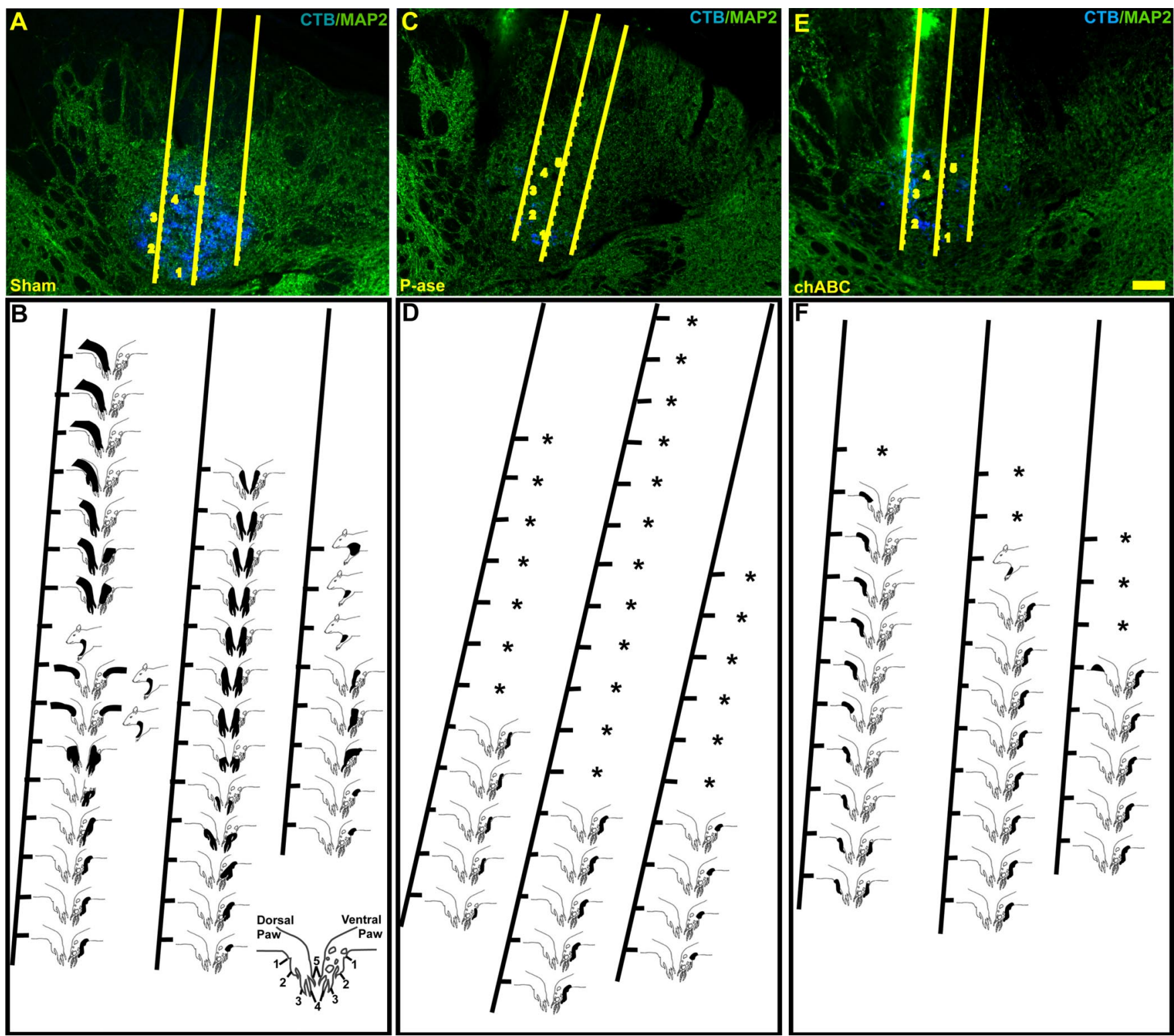

Figure 5. Images of representative sections through the cuneate nuclei and their matching receptive field maps from sham $(\boldsymbol{A}, \boldsymbol{B}), \mathrm{P}$-ase-treated $(\boldsymbol{C}, \boldsymbol{D})$ or chABC-treated $(\boldsymbol{E}, \boldsymbol{F})$ rats. MAP2-IR (green) and (TB-IR (blue) demonstrate the anatomical features and traced terminal field locations in relationship to the recording site locations observed along the microelectrode trajectory. Activity elicited by stimulation to the ipsilateral body surfaces revealed an orderly progression of receptive fields (black) dominated by areas around each of the digits and forepaw in the non-injured rats ( $\boldsymbol{B}$ ). After the C6-C7 spinal cord injury, the receptive fields were limited primarily to the digits 1 and 2 in both the P-ase-treated $(\boldsymbol{D})$ and chABC-treated $(\boldsymbol{F})$ injured rats. However, in the chABC-treated injured rats, these receptive fields were far more common (see Fig. 6) and could be mapped well into ectopic locations in the cuneate nucleus often located adjacent to areas of (TB-IR ( $\boldsymbol{E}$, $\boldsymbol{F})$. Silent areas $\left({ }^{*}\right)$, in which no activity could be elicited by stimulation to any body surface, were seen in both groups of injured rats but were far more common in the P-ase-treated rats $(\boldsymbol{D}, \boldsymbol{F}$; see Fig. $6 B)$. Numbers $1-5$ denote the normal locations of the digit terminal fields. Scale bar, $100 \mu \mathrm{m}$.

tinuous with digit 3 . There was a significant reduction (one-way ANOVA, $p<0.001$; post hoc Tukey's test, $p<0.001$ ) in the frequency of recording sites responding to forepaw stimulation $(46.3 \pm 3.3 \%)$ compared with the sham control rats (81.0 \pm $8.3 \%$ ) (Fig. 6A). Likewise, there was a significant increase (oneway ANOVA, $p<0.001$; post hoc Tukey's test, $p<0.001$ ) in the frequency of recording sites not responsive to any stimulus ( $\mathrm{P}$ ase, $36.2 \pm 8.6 \%$; sham, $5.6 \pm 1.2 \%$ ) (Fig. $6 B$ ). Collectively, these results demonstrate that the $\mathrm{C} 6-\mathrm{C} 7 \mathrm{SCI}$ significantly denervated the cuneate nucleus of functional primary afferent input from both the ipsilateral forepaw and other areas of the upper body.

The forepaw receptive fields encountered in injured rats that received chABC treatment were also restricted to the surfaces on and around digits 1,2, and a small portion of digit 3 (Figs. $5 E, F$, $6 C$ ). However, quite unlike the P-ase control animals (46.3 \pm $3.3 \%$ ), responses to forepaw stimulation in chABC-treated $\mathrm{SCI}$ rats were encountered with significantly greater frequency $(74.5 \pm 4.8 \%$; post hoc Tukey's test, $p<0.001$ ) (Fig. $6 A$ ). As was the case with the sham and P-ase-treated SCI rats, the recording sites responsive to digit and forepaw stimulation were often near areas dense with CTBimmunoreactive terminals (Fig. 5E). Much of the difference could be attributed to a significant expansion of digit 2 receptive fields throughout the cuneate nucleus (two-way ANOVA, $p<0.001$; post hoc Tukey's test, $p<0.001$ ) (Fig. $6 C$ ). Additionally, a significant ( post hoc Tukey's test, $p<0.05$ ) reduction in the number of unresponsive recording sites was observed in the chABC-treated rats 
A Forepaw Receptive Fields
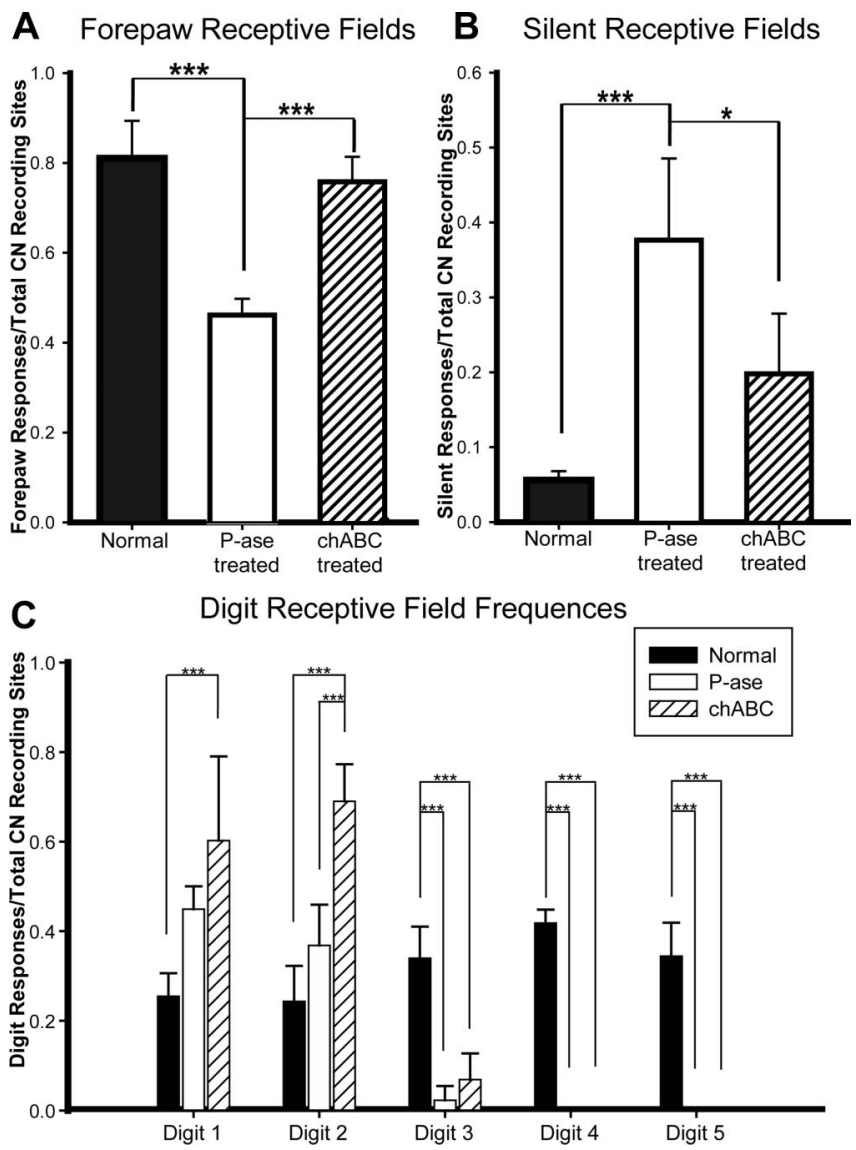

Figure 6. Quantification of receptive field frequencies from sham, P-ase-treated, and chABC-treated rats. The frequency of cuneate neuron activation in response to forepaw stimulation in the chABC-treated rats was significantly greater than that observed in P-ase-treated rats after $(6-C 7 \mathrm{SCl}(A)$. Additionally, there was a significant reduction in the number of recording sites in chABC-treated injured rats that could not be activated by any body surface when compared with $\mathrm{P}$-ase-treated injured rats $(\boldsymbol{B})$. Digits 1 and 2 receptive field frequencies were significantly increased in the chABC-treated injured rats but not in the $P$-ase-treated injured rats demonstrating that the expansion of territories from the primary afferents originating from these areas of the forepaw were responsible for much of the increases in cuneate activation (C). Data considered for statistical comparisons are shown by brackets: ${ }^{*} p<0.05 ;{ }^{* * *} p<0.001$.

$(19.1 \pm 7.5 \%)$ compared with the P-ase-treated rats $(36.2 \pm 8.6 \%)$ (Fig. 6B). Collectively, these results demonstrate that chABC treatment facilitated the spreading of receptive field territories from the uninjured primary afferents and suggest that the increase of CTB forepaw primary afferent terminals in the cuneate nucleus after chABC treatment reflects a functional expansion of their territories.

\section{Discussion}

We investigated whether chABC applied to the partially denervated adult rat cuneate nucleus reduced inhibition by CSPGs in the perineuronal net and promoted sprouting of the remaining forelimb primary afferents. Here we report that chABC, but not $\mathrm{P}$-ase, treatment effectively (1) reduced CSPGs within and surrounding the parenchyma of the cuneate nucleus, (2) resulted in a significantly increased area within nucleus cuneatus that was occupied by the remaining forepaw primary afferents, and (3) produced a significantly increased number of neurons within the cuneate nucleus that responded physiologically to the remaining afferents. We conclude from these results that reducing CSPGmediated inhibition within the cuneate nucleus markedly en- hances functional sprouting by spared primary afferents after cervical spinal cord dorsal column injury.

\section{Could the anatomical and functional plasticity induced by chABC be the result of lesion variability?}

In our model, lesion variability would have been manifested either by a cut that failed to transect the entire depth of the cuneate

fasciculus or a lesion complete in depth but in the wrong location. Both of these scenarios would have produced obvious discontinuous patterns of CTB-immunoreactive terminals in the cuneate nucleus and/or discontinuous receptive fields in these animals. These contentions are supported by the findings in one rat in which inspections of spinal cord sections revealed a C6 - C7 spinal cord lesion that did not completely transect the cuneate fasciculus. The pattern of CTB-IR in the cuneate nucleus was unique to this rat, and the animal was subsequently removed from the study. Furthermore, most of the increase in receptive field responses observed in the chABC-treated injured rats was from digit 2, and there was no significant difference between the responses observed from the little remaining input of digit 3 in the $\mathrm{P}$-ase-treated and the chABC-treated injured rats included in the study. Additionally, no responses to receptive fields of dermatomes supplied by dorsal roots entering the spinal cord caudal to $\mathrm{C} 6$ were observed in any of the rats studied. Had the increase in receptive field responses observed in the chABC-treated injured rats been attributable to lesion variability, we would have expected to see significant increases in the size or frequency of the digit 3 receptive fields or the presence of isolated and novel receptive fields from remaining digits or from other body surfaces caudal to those found in the $\mathrm{P}$-ase-treated injured rats.

\section{Effect of CSPGs and chABC on plasticity}

The precise role of matrix CSPGs in the regulation of plasticity and synapse formation is poorly understood (Steindler, 1993; Rhodes and Fawcett, 2004; Sandvig et al., 2004; Silver and Miller, 2004). The observations that CSPGs increase in areas of decreasing plasticity during development (Kalb and Hockfield, 1990a,b; Lander et al., 1997; Pizzorusso et al., 2002) and that their removal reactivates plasticity (Pizzorusso et al., 2002; Corvetti and Rossi, 2005) suggests an important contribution. It is likely that much of the matrix CSPG influence in the perineuronal net is attributable to its inhibition of terminal axonal outgrowth. Application of chABC has been demonstrated to reduce CSPG-related inhibition and facilitate axon regeneration in the injured spinal cord (Bradbury at al, 2002; Yick et al., 2003), through the dorsal root entry zone (Steinmetz et al., 2005), into peripheral nerve grafts (Yick et al., 2000), and through Schwann cell seeded channels and grafts (Chau et al., 2004; Fouad et al., 2005). Axon regeneration and sprouting into denervated areas of the striatum (Moon et al., 2001) and the superior colliculus (Tropea et al., 2003) were similarly facilitated by chABC application. Importantly, in several of the ChABC treatment studies, apparent synapse formation (Tropea et al., 2003; Steinmetz et al., 2005) and/or improved function were reported (Bradbury et al., 2002; Caggiano et al., 2005; Steinmetz et al., 2005). Our findings suggest that new synaptic contacts are made by sprouting forepaw primary afferents in the partially denervated cuneate nucleus after removal of extracellular matrix CSPGs. These results demonstrate, for the first time, a functional change directly linked to anatomical evidence of sprouting by spinal cord primary afferents after chABC treatment.

It has been hypothesized that perineuronal net CSPGs form a barrier between advancing axons and potential postsynaptic tar- 
gets (Yamaguchi, 2000). The first stages of synapse formation partially depend on the trafficking of secreted factors and interactions between adhesion molecules expressed on the surfaces of both presynaptic and postsynaptic membranes (Matus et al., 2000; Cohen-Cory, 2002; Vicario-Abejon et al., 2002). It is known that CSPGs can interfere with adhesive interactions and that such interruptions of ligand binding can be reduced by chABC digestion (Grumet et al., 1996; Milev et al., 1996). Additionally, it is likely that the highly charged glycosaminoglycan side chains interfere with diffusion of molecules secreted by both axons and dendrites (Gruskin et al., 2003). Some of the effects of chABC might be to increase interactions between surface proteins on the sprouting axons and open/vacant synaptic sites, thereby increasing the likelihood of synapse formation.

What are the precise cellular mechanisms that control and direct the axon terminal as it shifts positions once the perineuronal net is degraded? Although this remains a mystery in the CNS, observations on plastic changes at the neuromuscular junction suggest that the glia may play a role. It has been demonstrated that reinnervating motor axon terminals are guided to their targets by bridges of growth-promoting extracellular matrix found along the tips of Schwann cell processes that lead the advance of the axon (Ko and Chen, 1996). Similar interactions between astrocytes and the CNS extracellular matrix during synapse formation have also been suggested (Slezak and Pfrieger, 2003; Allen and Barres 2005). For instance, it is known that both thrombospondins 1 and 2, large glycoproteins produced by astrocytes that increase synapse formation (Christopherson et al., 2005), contain CSPG binding sites (Ehlers, 2005). The removal of CSPGs by chABC, in addition to freeing the axon from its cocoon, may also release the glia from theirs, thereby facilitating the synapse-promoting actions of the growth-promoting molecules that they also make.

Other mechanisms unrelated to overt sprouting or synapse formation but more to do with the efficacy or regulation of synaptic transmission may also be involved in the functional improvement observed after incomplete injuries. Partial removal of input into the cuneate nucleus results in reductions of lateral inhibition and subsequent increases of receptive fields from the intact inputs (Xu and Wall, 1996; Schwark et al., 1999). In our experiments, this may have contributed to the small but insignificant increase in the extent of the remaining receptive fields in the $\mathrm{P}$-ase-treated SCI rats. Furthermore, it has been shown in primate studies that previously nonfunctional axons poorly identified by CTB tracing are unmasked and contribute to injuryinduced plasticity by increasing both their conductivity and their terminal fields (Darian-Smith, 2004). In these studies, this process took many months. Whether chABC treatment could have potentiated these other types of plastic phenomena or awakened silent synapses is unknown, but such underappreciated potential effects of chABC cannot be excluded using the currently available methods.

\section{Does collateral sprouting improve forelimb function?}

In our experiments, wide areas of the once denervated cuneate nucleus were reactivated by primary afferents from restricted portions of the forepaw (digits 1 and 2). It is difficult to predict whether this maladjustment of the normal somatotopic map can promote improved forelimb function. In primates, relatively smaller ectopic distributions of remaining forelimb afferents in the cuneate nuclei are seen after very long periods of recovery after denervating injuries (Darian-Smith, 2004). It was demonstrated that these animals learn to adapt newly emerging sensory receptive fields to guide fine forelimb and digit movements requiring significant dexterity (Darian-Smith and Ciferri, 2005). Because rodents possess more limited forepaw acuity than that observed in primates, devising a method that reliably detects the effect of chABC on forepaw function will be challenging and the subject of future investigations.

\section{Conclusion}

Meaningful repair of axonal pathways after traumatic CNS injury is likely to involve the implementation of combined strategies. It has been demonstrated that the effects of chABC treatment on target reinnervation and sprouting are augmented by neurotrophins (Tropea et al., 2003) or when used with other strategies that increase the intrinsic regenerative capacity of injured axons (Steinmetz et al., 2005). Additional increases in synaptic plasticity might be seen by combining chABC with other approaches that increase regeneration and synapse formation. For example, treatments that relieve the axon of its envelope of growth inhibition brought about by myelin also lead to axonal sprouting, but here the typical response appears to be more far ranging than after chABC administration (Buffo et al., 2000, Li et al., 2004; Corvetti and Rossi, 2005). The combined use of altering myelin inhibitors or manipulation of the cAMP pathway (Filbin, 2003) may increase the synaptic plasticity observed after application of chABC. These combined approaches applied at target sites during axon regeneration or when used together with rehabilitative strategies designed to increase CNS reorganization (Edgerton et al., 2004) could result in improved functional recovery.

\section{References}

Allen NJ, Barres BA (2005) Signaling between glia and neurons: focus on synaptic plasticity. Curr Opin Neurobiol 15:542-548.

Bradbury EJ, Moon LD, Popat RJ, King VR, Bennett GS, Patel PN, Fawcett JW, McMahon SB (2002) Chondroitinase ABC promotes functional recovery after spinal cord injury. Nature 416:636-640.

Bruckner G, Bringmann A, Hartig W, Koppe G, Delpech B, Brauer K (1998) Acute and long-lasting changes in extracellular-matrix chondroitinsulphate proteoglycans induced by injection of chondroitinase $\mathrm{ABC}$ in the adult rat brain. Exp Brain Res 121:300-310.

Buffo A, Zagrebelsky M, Huber AB, Skerra A, Schwab ME, Strata P, Rossi F (2000) Application of neutralizing antibodies against NI-35/250 myelinassociated neurite growth inhibitory proteins to the adult rat cerebellum induces sprouting of uninjured purkinje cell axons. J Neurosci 20:2275-2286.

Caggiano AO, Zimber MP, Ganguly A, Blight AR, Gruskin EA (2005) Chondroitinase ABCI improves locomotion and bladder function following contusion injury of the rat spinal cord. J Neurotrauma 22:226-239.

Chau CH, Shum DK, Li H, Pei J, Lui YY, Wirthlin L, Chan YS, Xu XM (2004) Chondroitinase ABC enhances axonal regrowth through Schwann cellseeded guidance channels after spinal cord injury. FASEB J 18:194-196.

Christopherson KS, Ullian EM, Stokes CC, Mullowney CE, Hell JW, Agah A, Lawler J, Mosher DF, Bornstein P, Barres BA (2005) Thrombospondins are astrocyte-secreted proteins that promote CNS synaptogenesis. Cell 120:421-433.

Cohen-Cory S (2002) The developing synapse: construction and modulation of synaptic structures and circuits. Science 298:770-776.

Corvetti L, Rossi F (2005) Degradation of chondroitin sulfate proteoglycans induces sprouting of intact Purkinje axons in the cerebellum of the adult rat. J Neurosci 25:7150-7158.

Darian-Smith C (2004) Primary afferent terminal sprouting after a cervical dorsal rootlet section in the macaque monkey. J Comp Neurol 470:134-150.

Darian-Smith C, Ciferri MM (2005) Loss and recovery of voluntary hand movements in the macaque following a cervical dorsal rhizotomy. J Comp Neurol 491:27-45.

Davies SJ, Goucher DR, Doller C, Silver J (1999) Robust regeneration of adult sensory axons in degenerating white matter of the adult rat spinal cord. J Neurosci 19:5810-5822. 
Edgerton VR, Tillakaratne NJ, Bigbee AJ, de Leon RD, Roy RR (2004) Plasticity of the spinal neural circuitry after injury. Annu Rev Neurosci 27:145-167.

Ehlers MD (2005) Synapse formation: astrocytes spout off. Curr Biol 15:R134-R137.

Filbin MT (2003) Myelin-associated inhibitors of axonal regeneration in the adult mammalian CNS. Nat Rev Neurosci 4:703-713.

Fouad K, Schnell L, Bunge MB, Schwab ME, Liebscher T, Pearse DD (2005) Combining Schwann cell bridges and olfactory-ensheathing glia grafts with chondroitinase promotes locomotor recovery after complete transection of the spinal cord. J Neurosci 25:1169-1178.

Golgi C (1893) Intorno all'origine del quarto nervo cerebrale e una questione isto-fisiologica che a questo argomento si collega. Rendiconti della Reale Accademia Del Lincei 433-450.

Grumet M, Friedlander DR, Sakurai T (1996) Functions of brain chondroitin sulfate proteoglycans during development: interactions with adhesion molecules. Perspect Dev Neurobiol 3:319-330.

Gruskin EA, Iaci JP,Tseng JL, Caggiano AO (2003) Chondroitinase ABC I impacts diffusion rates in CNS tissue. Soc Neurosci Abstr 29:745.10.

Hubscher CH, Berkley KJ (1994) Responses of neurons in caudal solitary nucleus of female rats to stimulation of vagina, cervix, uterine horn and colon. Brain Res 664:1-8.

Jain N, Florence SL, Kaas JH (1995) Limits on plasticity in somatosensory cortex of adult rats: hindlimb cortex is not reactivated after dorsal column section. J Neurophysiol 73:1537-1546.

Jones LL, Margolis RU, Tuszynski MH (2003) The chondroitin sulfate proteoglycans neurocan, brevican, phosphacan, and versican are differentially regulated following spinal cord injury. Exp Neurol 182:399-411.

Kalb RG, Hockfield S (1990a) Induction of a neuronal proteoglycan by the NMDA receptor in the developing spinal cord. Science 250:294-296.

Kalb RG, Hockfield S (1990b) Large diameter primary afferent input is required for expression of the Cat-301 proteoglycan on the surface of motor neurons. Neuroscience 34:391-401.

Ko CP, Chen L (1996) Synaptic remodeling revealed by repeated in vivo observations and electron microscopy of identified frog neuromuscular junctions. J Neurosci 16:1780-1790.

Lander C, Kind P, Maleski M, Hockfield S (1997) A family of activitydependent neuronal cell-surface chondroitin sulfate proteoglycans in cat visual cortex. J Neurosci 17:1928-1939.

Li S, Liu BP, Budel S, Li M, Ji B, Walus L, Li W, Jirik A, Rabacchi S, Choi E, Worley D, Sah DW, Pepinsky B, Lee D, Relton J, Strittmatter, S. M (2004) Blockade of Nogo-66, myelin-associated glycoprotein, and oligodendrocyte myelin glycoprotein by soluble Nogo-66 receptor promotes axonal sprouting and recovery after spinal injury. J Neurosci 24:10511-10520.

Maslany S, Crockett DP, Egger MD (1991) Somatotopic organization of the dorsal column nuclei in the rat: transganglionic labelling with B-HRP and WGA-HRP. Brain Res 564:56-65.

Maslany S, Crockett DP, Egger MD (1992) The cuneate nucleus in the rat does have an anatomically distinct middle region. Neurosci Lett 139:130-134.

Massey JM, Bickford ME, Zhang YP, Giamarra B, Shields CB, Roisen FJ, Magnuson DSK, Onifer SM (2003) Vesicular glutamate transporter 1 as an anatomical marker of adult rat dorsal column axon synapse formation. J Rehabil Res Dev 40:45.

Massey JM, Matthews RT, Viapiano MS, Kalyfa A, Wagoner M, Decker JA, Cooper NGF, Onifer SM (2005) Chondroitin sulfate proteoglycan expression within the adult rat brainstem dorsal column nuclei following cervical spinal cord injury. Soc Neurosci Abstr 31:836.8.

Matthews RT, Kelly GM, Zerillo CA, Gray G, Tiemeyer M, Hockfield S (2002) Aggrecan glycoforms contribute to the molecular heterogeneity of perineuronal nets. J Neurosci 22:7536-7547.

Matus A, Brinkhaus H, Wagner U (2000) Actin dynamics in dendritic spines: a form of regulated plasticity at excitatory synapses. Hippocampus 10:555-560.

McKeon RJ, Schreiber RC, Rudge JS, Silver J (1991) Reduction of neurite outgrowth in a model of glial scarring following CNS injury is correlated with the expression of inhibitory molecules on reactive astrocytes. J Neurosci 11:3398-3411.

McKeon RJ, Jurynec MJ, Buck CR (1999) The chondroitin sulfate proteoglycans neurocan and phosphacan are expressed by reactive astrocytes in the chronic CNS glial scar. J Neurosci 19:10778-10788.

Meyer-Puttlitz B, Milev P, Junker E, Zimmer I, Margolis RU, Margolis RK
(1995) Chondroitin sulfate and chondroitin/keratan sulfate proteoglycans of nervous tissue: developmental changes of neurocan and phosphacan. J Neurochem 65:2327-2337.

Milev P, Maurel P, Haring M, Margolis RK, Margolis RU (1996) TAG-1/ axonin-1 is a high-affinity ligand of neurocan, phosphacan/proteintyrosine phosphatase-zeta/beta, and N-CAM. J Biol Chem 271:15716-15723.

Moon LD, Asher RA, Rhodes KE, Fawcett JW (2001) Regeneration of CNS axons back to their target following treatment of adult rat brain with chondroitinase ABC. Nat Neurosci 4:465-466.

Onifer SM, Whittemore SR, Holets VR (1993) Variable morphological differentiation of a raphe-derived neuronal cell line following transplantation into the adult rat CNS. Exp Neurol 122:130-142.

Onifer SM, Zhang YP, Burke DA, Brooks DL, Decker JA, McClure NJ, Floyd AR, Hall J, Proffitt BL, Shields CB, Magnuson DS (2005) Adult rat forelimb dysfunction after dorsal cervical spinal cord injury. Exp Neurol 192:25-38.

Pindzola RR, Doller C, Silver J (1993) Putative inhibitory extracellular matrix molecules at the dorsal root entry zone of the spinal cord during development and after root and sciatic nerve lesions. Dev Biol 156:34-48.

Pizzorusso T, Medini P, Berardi N, Chierzi S, Fawcett JW, Maffei L (2002) Reactivation of ocular dominance plasticity in the adult visual cortex. Science 298:1248-1251.

Rhodes KE, Fawcett JW (2004) Chondroitin sulphate proteoglycans: preventing plasticity or protecting the CNS? J Anat 204:33-48.

Sandvig A, Berry M, Barrett LB, Butt A, Logan A (2004) Myelin-, reactive glia-, and scar-derived CNS axon growth inhibitors: expression, receptor signaling, and correlation with axon regeneration. Glia 46:225-251.

Schwark HD, Tennison CF, Ilyinsky OB, Fuchs JL (1999) Inhibitory influences on receptive field size in the dorsal column nuclei. Exp Brain Res 126:439-442.

Silver J, Miller JH (2004) Regeneration beyond the glial scar. Nat Rev Neurosci 5:146-156.

Slezak M, Pfrieger FW (2003) New roles for astrocytes: regulation of CNS synaptogenesis. Trends Neurosci 26:531-535.

Snow DM, Steindler DA, Silver J (1990) Molecular and cellular characterization of the glial roof plate of the spinal cord and optic tectum: a possible role for a proteoglycan in the development of an axon barrier. Dev Biol 138:359-376.

Steindler DA (1993) Glial boundaries in the developing nervous system. Annu Rev Neurosci 16:445-479.

Steinmetz MP, Horn KP, Tom VJ, Miller JH, Busch SA, Nair D, Silver DJ, Silver J (2005) Chronic enhancement of the intrinsic growth capacity of sensory neurons combined with the degradation of inhibitory proteoglycans allows functional regeneration of sensory axons through the dorsal root entry zone in the mammalian spinal cord. J Neurosci 25:8066-8076.

Takahashi-Iwanaga H, Murakami T, Abe K (1998) Three-dimensional microanatomy of perineuronal proteoglycan nets enveloping motor neurons in the rat spinal cord. J Neurocytol 27:817-827.

Tang X, Davies JE, Davies SJ (2003) Changes in distribution, cell associations, and protein expression levels of NG2, neurocan, phosphacan, brevican, versican $\mathrm{V} 2$, and tenascin-C during acute to chronic maturation of spinal cord scar tissue. J Neurosci Res 71:427-444.

Tropea D, Caleo M, Maffei L (2003) Synergistic effects of brain-derived neurotrophic factor and chondroitinase $\mathrm{ABC}$ on retinal fiber sprouting after denervation of the superior colliculus in adult rats. J Neurosci 23:7034-7044.

Vicario-Abejon C, Owens D, McKay R, Segal M (2002) Role of neurotrophins in central synapse formation and stabilization. Nat Rev Neurosci 3:965-974.

Xu J, Wall JT (1996) Cutaneous representations of the hand and other body parts in the cuneate nucleus of a primate, and some relationships to previously described cortical representations. Somatosens Mot Res 13:187-197.

Yamaguchi Y (2000) Lecticans: organizers of the brain extracellular matrix. Cell Mol Life Sci 57:276-289.

Yick LW, Wu W, So KF, Yip HK, Shum DK (2000) Chondroitinase ABC promotes axonal regeneration of Clarke's neurons after spinal cord injury. NeuroReport 11:1063-1067.

Yick LW, Cheung PT, So KF, Wu W (2003) Axonal regeneration of Clarke's neurons beyond the spinal cord injury scar after treatment with chondroitinase ABC. Exp Neurol 182:160-168. 